Cristine Bonfim 1

Fábio Lessa 2

Conceição Oliveira ${ }^{3}$

Maria José Evangelista 4

Marlene do Espírito Santo 3

Elizeide Meireles 5

José Costa Pereira 6

Zulma Medeiros 3,7

\section{Situação da filariose bancroftiana na Região Metropolitana do Recife: estudo em uma área endêmica no Município de Jaboatão dos Guararapes, Pernambuco, Brasil}

\author{
The occurrence and distribution of lymphatic \\ filariasis in Greater Metropolitan Recife: \\ the case of an endemic area in Jaboatão \\ dos Guararapes, Pernambuco, Brazil
}

1 Departamento de Saúde Coletiva, Centro de Pesquisas Aggeu Magalhães, Fundação Oswaldo Cruz. Av. Moraes Rego s/n, Recife, PE 50670-420, Brasil. crisbonfim@aol.com 2 Centro de Ciências da Saúde, Universidade Federal de Pernambuco. Av. Moraes Rego $s / n$, Recife, PE 50670-420, Brasil.

3 Serviço de Referência Nacional em Filariose, Departamento de Parasitologia, Centro de Pesquisas Aggeu Magalhães, Fundação Oswaldo Cruz. Av. Moraes Rego s/n, Recife, PE 50670-420, Brasil. 4 Instituto Materno Infantil de Pernambuco. Rua dos Coelhos 300, Recife, PE 50070-550, Brasil. 5 Secretaria de Saúde Municipal de Jaboatão dos Guararapes. Av. General Barreto de Menezes 1648, Jaboatão dos Guararapes, $P E$ 54410-100, Brasil.

6 Fundação Nacional de Saúde. Av. Rosa e Silva 1489, Recife, PE 52050-020, Brasil.

7 Departamento de

Patologia, Instituto de Ciências Biológicas, Universidade de Pernambuco. Rua Anóbio Marques 310 , Recife, PE 50100-130, Brasil.

\begin{abstract}
This article analyzes the results of an epidemiological survey on the occurrence and distribution of lymphatic filariasis in Cavaleiro, a district in the city of Jaboatão dos Guararapes in Greater Metropolitan Recife, Pernambuco State, Brazil. The district was divided into 12 areas, and a cross-sectional technique was used to examine 9,520 individuals, of whom 2.2\% were found to be infected. There were positive cases in 11 of the areas. The prevalence rate ranged from $0 \%$ to 5.15\%. The majority (64.3\%) of infected individuals were males $(p<0.05)$. Infected individuals were identified in all age groups. The results show that lymphatic filariasis is still an important public health problem in the Cavaleiro district and that it deserves attention by municipal authorities and health planners.
\end{abstract}

Key words Filariasis; Wuchereria bancrofti; Morbidity Surveys

Resumo Este artigo apresenta os resultados do inquérito epidemiológico que estudou a ocorrência e a distribuição da filariose bancroftiana no Distrito de Cavaleiro, Município de Jaboatão dos Guararapes, Pernambuco. O desenho de estudo empregado foi o seccional. Foram analisados 9.520 indivíduos da população residente nos 12 bairros que compõem o distrito. Deste total, detectou-se 213 microfilarêmicos (2,2\%). Cerca de 91,7\% dos bairros pesquisados apresentaram casos de infecção filarial, com prevalências variando de $0 \%$ a 5,15\%. A população masculina foi a mais acometida pela infecção, com 64,3\% dos casos ( $p<0,05)$. Verificou-se, também, a existência de positividade em todas as faixas etárias examinadas. Esses resultados indicam que a filariose bancroftiana permanece como um problema de saúde pública na região, necessitando de medidas de controle e atenção dos administradores e planejadores de saúde.

Palavras-chave Filariose; Wuchereria bancrofti; Inquéritos de Morbidade 


\section{Introdução}

A filariose linfática causada pela Wuchereria bancrofti é considerada um grave problema de saúde pública em várias regiões do mundo. Estima-se que existam 1,2 bilhão de pessoas residentes em áreas endêmicas, isto equivale a 1/5 da população mundial (Dean, 2000). Dessas, cerca de 120 milhões já estão infectadas (Michael \& Bundy, 1997; Michael et al., 1996; WHO, 2000).

No Brasil, a partir da criação da campanha contra a filariose linfática pelo Ministério da Educação e Saúde, em 1951, foram efetuados inquéritos epidemiológicos em 538 localidades (1951 a 1958), sendo encontrados portadores de microfilaremia em $89(16,5 \%)$ das mesmas. No entanto, em apenas 11 (Manaus, Amazonas 0,2\%; Belém, Pará - 9,8\%; São Luís, Maranhão 0,6\%; Recife, Pernambuco - 6,9\%; Maceió, Alagoas $-0,3 \%$; Salvador, Bahia $-0,4 \%$; Castro Alves, Bahia - 5,9\%; Florianópolis, Santa Catarina - 1,4\%; Ponta Grossa, Paraná - 14,5\%; Barra de Laguna, Santa Catarina - 9,4\%; e Porto Alegre, Rio Grande do Sul - 0,1\%) dessas localidades ficou comprovada a transmissão ativa por meio do encontro simultâneo de mosquitos com larvas infectadas e indivíduos parasitados (Franco \& Lima, 1967; Rachou, 1957, 1960; Rachou et al., 1956).

Sucessivamente aos inquéritos, foram realizadas as ações de controle da filariose linfática que consistiam, basicamente, de duas linhas de ações: tratamento do indivíduo parasitado e, quando necessário, o combate ao vetor com aplicação de inseticidas químicos (Franco \& Lima, 1967). Essas medidas levaram a uma considerável redução nas taxas de prevalência, sendo quase todos os focos extintos (MS, 1985).

Atualmente, permanecem como focos da endemia a Região Metropolitana do Recife, Pernambuco (RMR), Maceió, Alagoas e Belém, Pará, estimando-se em 49 mil o número de infectados (MS, 2000; WHO, 2000).

$\mathrm{Na}$ RMR foram realizados diversos estudos (Albuquerque, 1993; Albuquerque et al., 1995; Medeiros et al., 1992, 1999), tanto em áreas reconhecidamente endêmicas, quanto em áreas indenes. No Recife, foram encontradas taxas de prevalência de até $15 \%$ de microfilarêmicos (Maciel et al., 1994), em alguns bairros carentes.

Um estudo desenvolvido nos três principais municípios da RMR (Recife, Olinda e Jaboatão dos Guararapes), comprovou a existência de transmissão ativa, por meio do encontro de casos de infecções autóctones e do índice de infectividade vetorial. Essas constatações evidenciaram a fixação e a expansão da endemia na RMR (Medeiros et al., 1992).
Estudo posterior, examinando 23.773 soldados do Exército Brasileiro, oriundos de vários bairros da RMR (1989 a 1995), revelou a existência de casos autóctones de infecção filarial em sete municípios da RMR, três reconhecidamente endêmicos (Jaboatão dos Guararapes, Olinda e Recife) e quatro de endemicidade recente (Paulista, Abreu e Lima, Cabo de Santo Agostinho e Camaragibe) (Medeiros et al., 1999).

Considerando tais indícios sobre a ocorrência da filariose bancroftiana em Jaboatão dos Guararapes, foi realizado um inquérito epidemiológico visando descrever a distribuição da parasitose no distrito de Cavaleiro, área de maior densidade populacional e expansão territorial desse município, reconhecendo-se essa como medida preliminar à implantação de qualquer estratégia de controle da filariose bancroftiana.

\section{Materiais e métodos}

A área de estudo corresponde ao Distrito de Cavaleiro, Município de Jaboatão dos Guararapes, localizado na RMR, a $18 \mathrm{~km}$ da capital do estado, com 137.826 habitantes, distribuídos em 27.969 domicílios particulares (IBGE, 1996).

Foi definido como desenho de estudo o seccional de base populacional em função da necessidade de se verificar a ocorrência da microfilaremia no Distrito de Cavaleiro, uma vez que não se dispõe de dados sobre filariose nos sistemas de informações de abrangência nacional.

As variáveis de análise foram agrupadas em dependentes (presença de microfilaremia segundo o exame de gota espessa) e independentes (sexo, faixa etária, bairro de residência na ocasião do inquérito e relato de manifestações clínicas). Essas variáveis foram obtidas por meio da aplicação de um questionário fechado contendo informações individuais, relacionadas a doença filarial (uso prévio de tratamento específico e relatos clínicos sugestivos da doença) e do exame da gota espessa.

Os dados populacionais foram constituídos com base em amostra do universo de 27.969 domicílios, estimando-se um percentual de positividade para os domicílios de $6 \%$ de filariose nas áreas estudadas e um erro aceitável de $5 \%$ para cada intervalo de confiança (IC) de 95\%. Chegando-se a um quantitativo de 2.011 domicílios.

O cadastramento dos domicílios foi realizado, segundo a distribuição dos setores censitários que compõem o distrito (137), para que a toda a área territorial fosse trabalhada na investigação. Porém, três setores censitários fo- 
ram excluídos devido a ausência de habitações, obtendo-se um total de 134. Embora, fosse necessário o cadastramento de apenas 16 domicílios por setor censitário, foram cadastros vinte, considerando as possíveis recusas dos moradores em participar do inquérito.

A escolha desses vinte domicílios no setor foi realizada por meio de consulta ao mapa do setor censitário, manipulado com a definição de seus quadrantes e a aplicação de uma reta na diagonal (2o e 3o quadrantes), unindo-se os quadrantes opostos. Após a localização do ponto médio de cada um (2o e 3o) , foram selecionados dois logradouros, um em cada ponto médio do quadrante, sendo trabalhados dez domicílios em cada rua, totalizando 20 em cada setor censitário.

A coleta de material biológico foi realizada tendo como protocolo o indicado pela Organização Mundial da Saúde (OMS) para inquéritos populacionais (WHO, 1994). Consistindo a coleta de amostra biológica, mediante a retirada de aproximadamente 50 microlitros de sangue capilar, com material descartável, no horário das 23h à 1h (Dreyer et al., 1996), durante o qual foi confeccionada a gota espessa não mensurada. Após desemoglobinizada, as gotas espessas foram coradas com eosina e contracoradas com Giemsa. O controle de qualidade para a leitura das lâminas foi realizado em 100\% dos positivos e 30\% dos negativos (WHO, 1994).

Utilizou-se como critério para a inclusão dos indivíduos no estudo a sua autorização mediante a assinatura do termo de consentimento livre e esclarecido, estando automaticamente excluídos os que não o assinaram. Com a identificação dos casos de microfilaremia, foi assegurado o tratamento com citrato de dietilcarbamazina (DEC) na posologia recomendada pela OMS (WHO, 1994), cumprindo-se as exigências previstas na Resolução 01/88 do Conselho Nacional de Saúde (Decreto no 93.933 14/01/1987). Informações sobre os aspectos básicos da doença filarial foram dadas à população-alvo da pesquisa. Este estudo foi aprovado pela Comissão de Ética em Pesquisa do Centro de Pesquisas Aggeu Magalhães, Fundação Oswaldo Cruz (CPqAM, FIOCRUZ).

Para processamento dos dados foi utilizado o programa Epi Info 6.04b. Com o banco de dados estruturado, foram realizadas análises nos módulos Analysis, Statcalc e Epitable.

\section{Resultados}

Foram cadastradas 11.540 pessoas, residentes em 2.580 domicílios, distribuídas pelos 12 bair- ros que compõem o distrito. Dessas 11.540 pessoas, $1.102(9,5 \%)$ encontravam-se ausentes do domicílio na ocasião da coleta do material biológico e $918(8,0 \%)$ recusaram-se a realizar o exame. O total de pessoas das quais foram coletadas amostras de material biológico para a confecção da gota espessa foi de 9.520 (82,5\%).

Foram examinadas pessoas de ambos os sexos e a idade variou de 01 a 99 anos. Das 9.520 pessoas examinadas, 213 estavam infectadas, observando-se, portanto, uma taxa de prevalência média para o distrito de 2,2\%. Nos 134 setores censitários foram examinadas uma média de 71 pessoas (46-88). A média de habitantes por domicílio foi de 4,46, com variação de 1 a 18 habitantes. Em 166 (6,4\%) dos 2.580 domicílios estudados foram encontrados um ou mais indivíduos microfilarêmicos.

A distribuição da ocorrência da infecção segundo o bairro demonstrou positividade em 11 $(91,7 \%)$ dos 12 pesquisados. A amplitude das taxas nos bairros variou entre 0 a $5,1 \%$. Em dois desses bairros, a taxa de prevalência de microfilaremia foi superior à taxa de todo o distrito $(2,2 \%)$, conforme se observa na Tabela 1 e na Figura 1. Considerando-se somente esses dois bairros de maior endemicidade, a taxa de prevalência encontrada foi de $4,8 \%$.

A análise da distribuição do percentual de microfilarêmicos, segundo faixa etária e sexo, demonstra que entre os 10-14 anos de idade a prevalência da parasitose foi maior no sexo feminino, entretanto, esta diferença por sexo não foi estatisticamente significante $(p>0,05)$. Nas demais faixas etárias foram verificadas uma maior positividade para o sexo masculino, sendo esta diferença significativa em termos estatísticos no intervalo compreendido entre os 20 e 39 anos e $>60$ anos (Tabela 2). Nestas faixas de idade, o risco de estar parasitado entre os indivíduos do sexo masculino foi superior ao feminino. Conforme visto na Figura 2, nos dois sexos, as maiores prevalências foram encontradas entre a população jovem.

A população masculina mostrou-se mais acometida pela infecção, com 137 casos, representando $64,3 \%$ do total e taxa de prevalência de 3,3\%. Já a população feminina teve 76 casos positivos, o que equivale a $35,7 \%$ de todas as ocorrências, com taxa de prevalência de $1,4 \%$. As prevalências entre os sexos foram significativamente diferentes $\left(\chi^{2}=35,97 ; \mathrm{p}<0,05\right.$; Razão de prevalência: 2,30; IC95\%: 1,74-3,04). Conforme observado na Tabela 2.

No que concerne às queixas de manifestações clínicas (Tabela 3), observa-se que dos 213 casos positivos, $13(6,1 \%)$ relataram sinais ou sintomas clínicos e 200 (93,9\%) não relataram 
Tabela 1

Distribuição da população examinada e microfilarêmicos por Wuchereria bancrofti, segundo o bairro, no Distrito de Cavaleiro, Jaboatão dos Guararapes, Pernambuco, Brasil, 2002.

\begin{tabular}{|c|c|c|c|c|c|c|c|}
\hline Bairro & $\begin{array}{l}\text { Setor } \\
\text { censitário }\end{array}$ & $\begin{array}{l}\text { Número de } \\
\text { examinados }\end{array}$ & $\begin{array}{l}\text { Número de } \\
\text { positivos }\end{array}$ & $\begin{array}{l}\text { Taxa de } \\
\text { prevalência }\end{array}$ & RP & $\chi^{2}$ & Valor $p$ \\
\hline Cavaleiro & 30 & 2.384 & 123 & 5,15 & \multicolumn{3}{|c|}{ Grupo referência } \\
\hline Curado I & 6 & 231 & 4 & 1,73 & $2,98(1,05-9,55)$ & 4,29 & 0,03 \\
\hline Curado II & 6 & 437 & 1 & 0,22 & $22,55(3,42-43,05)$ & 19,09 & 0,00 \\
\hline Curado III & 7 & 502 & - & - & - & - & - \\
\hline Curado IV & 25 & 1.669 & 24 & 1,43 & $3,59(2,26-5,73)$ & 35,39 & 0,00 \\
\hline Ibura & 14 & 998 & 4 & 0,40 & $12,87(4,58-40,99)$ & 40,41 & 0,00 \\
\hline Lote Grande Recife & 6 & 348 & 7 & 2,01 & $2,56(1,15-6,04)$ & 5,52 & 0,01 \\
\hline Jardim Monte Verde & 7 & 439 & 12 & 2,73 & $1,89(1,01-3,62)$ & 3,92 & 0,04 \\
\hline Socorro & 5 & 307 & 6 & 1,95 & $2,64(1,11-6,69)$ & 5,03 & 0,02 \\
\hline Sucupira & 14 & 1.146 & 19 & 1,65 & $3,11(1,87-5,24)$ & 22,08 & 0,00 \\
\hline Vila Dois Carneiros & 7 & 477 & 2 & 0,42 & $12,31(2,99-72,11)$ & 19,10 & 0,00 \\
\hline Zumbi do Pacheco & 10 & 582 & 11 & 1,89 & $2,73(1,42-5,38)$ & 10,07 & 0,00 \\
\hline Total & 137 & 9.520 & 213 & 2,2 & - & - & - \\
\hline
\end{tabular}

RP = Razão de prevalência; $\chi^{2}=$ Teste Qui-quadrado.

Figura 1

Distribuição da população examinada e microfilarêmicos por Wuchereria bancrofti, segundo o bairro, no distrito de Cavaleiro, Jaboatão dos Guararapes, Pernambuco, Brasil, 2002.

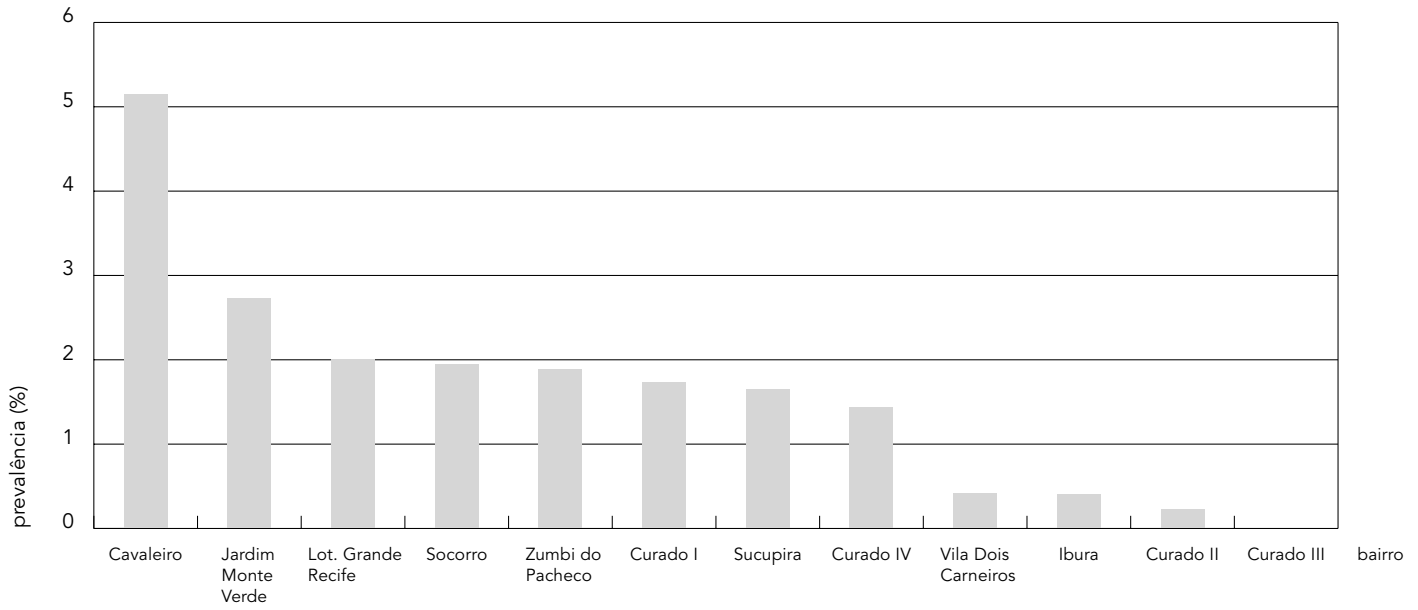


Tabela 2

Distribuição dos microfilarêmicos por Wuchereria bancrofti, segundo o sexo e a faixa etária,

no distrito de Cavaleiro, Jaboatão dos Guararapes, Pernambuco, Brasil, 2002.

\begin{tabular}{|c|c|c|c|c|c|c|c|c|c|}
\hline $\begin{array}{l}\text { Faixa } \\
\text { etária } \\
\text { (anos) }\end{array}$ & $\begin{array}{l}\text { Número de } \\
\text { examinados }\end{array}$ & $\begin{array}{l}\text { Masculino } \\
\text { Número de } \\
\text { positivos }\end{array}$ & Prevalência & $\begin{array}{l}\text { Número de } \\
\text { examinados }\end{array}$ & $\begin{array}{l}\text { Feminino } \\
\text { Número de } \\
\text { positivos }\end{array}$ & Prevalência & RP & $\chi^{2}$ & Valor $\mathrm{p}$ \\
\hline $1-9$ & 777 & 4 & 0,51 & 724 & 2 & 0,27 & $1,86(0,34-1014)$ & 0,1 & 0,74 \\
\hline $10-14$ & 558 & 14 & 2,50 & 591 & 17 & 2,87 & $0,87(0,43-1,75)$ & 0,04 & 0,8 \\
\hline $15-19$ & 508 & 17 & 3,34 & 577 & 13 & 2,25 & $1,49(0,73-3,03)$ & 0,83 & 0,36 \\
\hline $20-24$ & 403 & 24 & 5,95 & 510 & 11 & 2,15 & $2,76(1,37-5,57)$ & 7,81 & 0,00 \\
\hline $25-29$ & 321 & 16 & 4,98 & 460 & 5 & 1,07 & $4,63(1,71-12,50)$ & 9,67 & 0,00 \\
\hline $30-34$ & 309 & 12 & 3,88 & 463 & 4 & 0,86 & $4,50(1,46-13,81)$ & 6,9 & 0,00 \\
\hline $35-39$ & 285 & 19 & 6,66 & 448 & 7 & 1,56 & $4,27(1,82-10,02)$ & 11,81 & 0,00 \\
\hline $40-44$ & 231 & 3 & 1,29 & 330 & 2 & 0,60 & $2,14(0,36-12,72)$ & 0,16 & 0,68 \\
\hline $45-49$ & 203 & 6 & 2,95 & 309 & 5 & 1,61 & $1,83(0,56-5,91)$ & 0,5 & 0,47 \\
\hline $50-59$ & 305 & 10 & 3,27 & 471 & 6 & 1,27 & $2,57(0,95-7,01)$ & 2,76 & 0,09 \\
\hline $60+$ & 268 & 12 & 4,47 & 440 & 4 & 0,90 & $4,93(1,60-15,12)$ & 8,05 & 0,00 \\
\hline Ignorado & 13 & - & - & 16 & - & - & - & - & - \\
\hline Total & 4.181 & 137 & 3,27 & 5.339 & 76 & 1,42 & $2,30(1,74-3,04)$ & 35,97 & 0,00 \\
\hline
\end{tabular}

$\mathrm{RP}=$ Razão de prevalência; $\chi^{2}=$ Teste Qui-quadrado.

Figura 2

Distribuição dos microfilarêmicos por Wuchereria bancrofti, segundo o sexo e a faixa etária, no distrito de Cavaleiro, Jaboatão dos Guararapes, Pernambuco, Brasil, 2002.

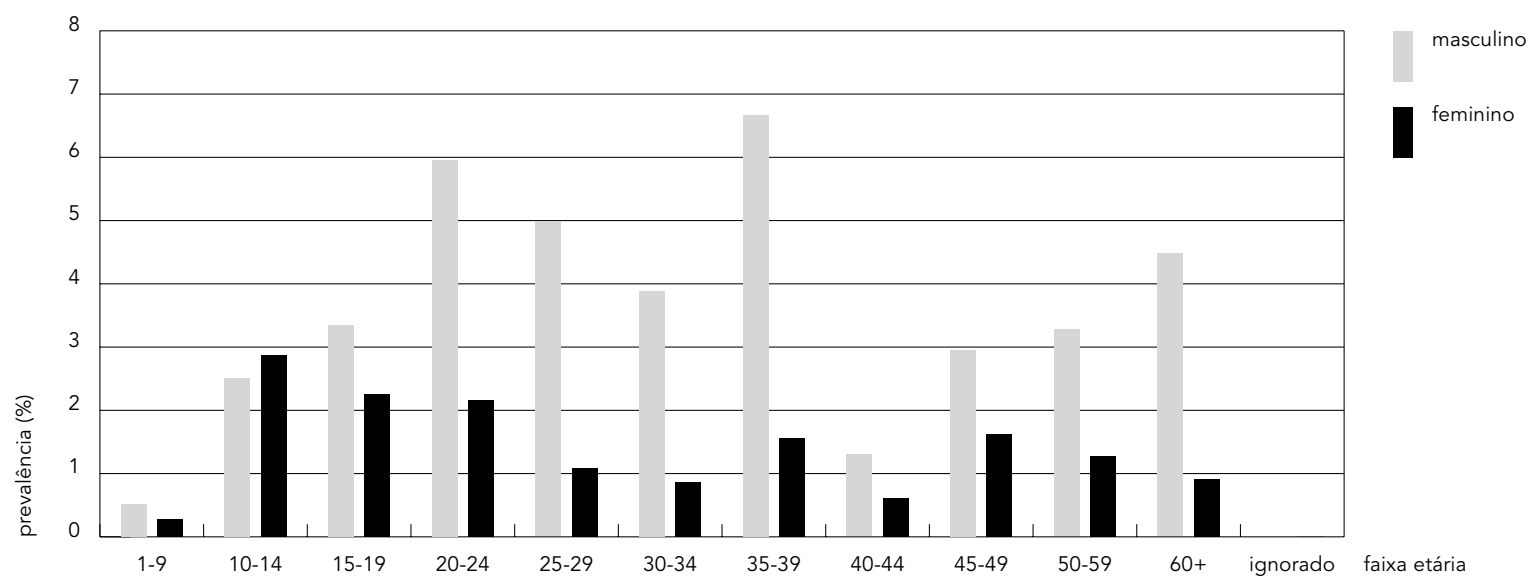


Tabela 3

Distribuição da população examinada para Wuchereria bancrofti, segundo os relatos de manifestações clínicas e o sexo, no distrito de Cavaleiro, Jaboatão dos Guararapes, Pernambuco, Brasil, 2002.

\begin{tabular}{|c|c|c|c|c|c|c|}
\hline \multirow[t]{2}{*}{ Clínica } & \multicolumn{2}{|c|}{ Masculino } & \multicolumn{2}{|c|}{ Feminino } & \multicolumn{2}{|c|}{ Total } \\
\hline & Amicrofilarêmicos & Microfilarêmicos & Amicrofilarêmicos & Microfilarêmicos & Amicrofilarêmicos & Microfilarêmicos \\
\hline Elefantíase & 4 & - & 3 & - & 7 & - \\
\hline Elefantíase e erisipela & - & - & 1 & - & 1 & - \\
\hline Erisipela & 61 & 2 & 185 & 3 & 246 & 5 \\
\hline Hidrocele & 110 & 2 & NA & NA & 110 & 2 \\
\hline Hidrocele e elefantíase & - & 1 & NA & NA & 1 & 1 \\
\hline Hidrocele e erisipela & 4 & - & NA & NA & 4 & - \\
\hline Hidrocele e varicocele & 2 & - & NA & NA & 2 & - \\
\hline Urina leitosa & 1 & - & 10 & - & 11 & - \\
\hline Varicocele & 17 & 1 & NA & NA & 17 & 1 \\
\hline Varicocele e erisipela & 1 & - & NA & NA & 1 & - \\
\hline Nenhum sinal & 3.766 & 127 & 4.947 & 72 & 8.713 & 199 \\
\hline Outros & 41 & 3 & 76 & 1 & 117 & 4 \\
\hline Ignorado & 37 & 1 & 41 & - & 78 & 1 \\
\hline Total & 4.044 & 137 & 5.263 & 76 & 9.307 & 213 \\
\hline
\end{tabular}

NA = não aplicado.

nenhum sintoma, verificando-se que a maior parte dos indivíduos infectados era composta por microfilarêmicos assintomáticos. Entre os indivíduos sintomáticos, nove $(69,2 \%)$ eram do sexo masculino e quatro $(30,8 \%)$ do sexo feminino, não sendo esta, contudo, uma diferença estatisticamente significante $(\mathrm{p}>0,05)$. O relato de manifestação clínica mais freqüente foi à erisipela com cinco casos $(38,5 \%)$.

\section{Discussão}

A filariose bancroftiana é considerada endêmica no Recife, desde os primeiros inquéritos realizados no Brasil em 1950 (Franco \& Lima, 1967; Rachou, 1960). Após a implementação das medidas de controle, acreditou-se que a endemia seria facilmente controlada e gradativamente extinta no país (MS, 1985). Contudo, a filariose permanece endêmica e em processo de expansão atingindo áreas consideradas indenes (Medeiros, 1998).

Os resultados do inquérito epidemiológico indicam a manutenção e a expansão da filariose na RMR. A taxa de prevalência identificada foi de 2,2\% para o distrito de Cavaleiro. Entretanto, a análise realizada segundo unidades menores como bairros, revelou uma variação da taxa de prevalência de $0 \%$ até $5,15 \%$. Segundo a classificação de risco da OMS (1988), as taxas de prevalência podem ser estratificadas em baixa (menor que 5\%), média (5 a 9\%) e alta (maior ou igual a 10\%); os dois primeiros tipos de risco foram encontrados na área estudada. Faz-se mister enfatizar a ampla distribuição da ocorrência da infecção na localidade, fato evidenciado por meio do encontro de positividade em $11(91,7 \%)$ dos 12 bairros examinados.

Outro indicativo da endemicidade da filariose no Distrito de Cavaleiro é o encontro de indivíduos infectados em todas as faixas etárias, especialmente entre os mais jovens (Dunyo et al., 1996; Rajasekariah et al., 1991). Observou-se que o maior risco de ter infecção filarial foi apresentado entre os indivíduos do sexo masculino, na faixa etária dos 25-29 anos. De fato, esse parece ser um padrão das manifestações da infecção, maior acometimento de indivíduos do sexo masculino, na faixa etária de 20 a 30 anos (Brabin, 1990).

Destaca-se o número de microfilarêmicos nas faixas etárias jovens, 10-19, 20-29 e 30-39 anos, encontrado-se 61, 56 e 42 indivíduos parasitados, respectivamente. Esses achados sugerem que a filariose é endêmica na região há bastante tempo, levando-se em consideração o longo período decorrido até o aparecimento da doença filarial. Quanto maior o número de indivíduos jovens infectados, maior é a transmissibilidade da parasitose na área (Das et al., 1990).

Esses resultados, quanto à distribuição por sexo, assemelham-se aos de outros estudos rea- 
lizados em diversas localidades do mundo. Pani et al. (1991) e Das et al. (1990), em pesquisas desenvolvidas na Índia, verificaram a existência de prevalências significativamente maiores entre os indivíduos do sexo masculino quando comparados com os femininos. Já Dunyo et al. (1996), em Gana, só verificaram essa diferença entre os sexos em duas das quatro aldeias pesquisadas, entretanto, observaram um aumento das taxas de prevalência proporcional ao aumento da idade.

No Recife, desde os primeiros inquéritos epidemiológicos para a detecção da prevalência da filariose linfática, conduzidos por Azevedo e Dobbin Jr. (1952) e Rachou et al. (1956), tem-se verificado uma prevalência maior da infecção filarial nos indivíduos do sexo masculino em comparação com o feminino.

Maciel et al. (1994), em estudo realizado em duas cidades da RMR (Olinda e Recife), identificaram uma maior prevalência entre os indivíduos do sexo masculino $(15,76 \%$ e $14,55 \%)$ em relação ao feminino (9,36\% e 12,65\%), indicando ser o sexo masculino fator predisponente para a infecção parasitária.

Albuquerque et al. (1995), estudando a prevalência da filariose bancroftiana em duas áreas (Coque e Mustardinha) da cidade do Recife, verificaram que, em ambas as localidades, o sexo masculino apresentou uma taxa de prevalência de microfilaremia superior à taxa feminina (Coque $14,1 \%$ e $7,8 \%$; Mustardinha $12,6 \%$ e 6,6\%).

Outro dado relevante sobre a endemicidade da filariose foi o relato de manifestações clínicas entre os indivíduos examinados. A apresentação clínica da filariose é bastante diversificada e depende, entre outros fatores, do estádio do parasito envolvido e da resposta imunológica do paciente (Dreyer \& Norões, 1997b).

Em áreas de endemicidade de bancroftose, a maioria dos indivíduos tem poucas manifestações clínicas, apesar da presença de microfilária circulante no sangue. Esse fato foi verificado no estudo ora apresentado: dos 213 casos de infecção filarial, 200 (93,3\%) eram de indivíduos microfilarêmicos assintomáticos.

Do ponto de vista epidemiológico, os indivíduos infectados e assintomáticos desempenham um importante papel na manutenção da transmissão da filariose, pois eles constituem a principal fonte de infecção do vetor (Medeiros, 1998).

Estudos desenvolvidos por Dreyer \& Norões (1997a), identificaram que cerca de $15 \%$ dos indivíduos infectados desenvolverão alguma forma de manifestação clínica. No inquérito epidemiológico que serve de base a este trabalho, $6,1 \%$ dos indivíduos infectados relataram alguma queixa relacionada com a filariose bancroftiana.

Contudo, é possível que os indivíduos aparentemente assintomáticos já apresentem alguma forma subclínica de manifestação da doença. Estudos utilizando a ultra-sonografia demonstraram a existência de vermes adultos localizados em linfáticos da área escrotal de adultos jovens assintomáticos (Amaral et al., 1994). Segundo a OMS, das 120 milhões de pessoas infectadas, 44 milhões têm sinais visíveis da doença - hidrocele ou linfedema - e outros 76 milhões têm manifestação pré-clínica (WHO, 2000).

A ocorrência das duas formas clínicas mais mencionadas pelos examinados, a hidrocele e a erisipela, suscitam a necessidade de um estudo clínico mais detalhado, que busque verificar a existência de associação com a infecção filarial. A detecção precoce das alterações linfáticas contribuiria sobremaneira para evitar possíveis problemas crônicos, muitas vezes irreversíveis e estigmatizantes.

Os resultados deste trabalho fornecem informações relevantes para o planejamento das ações de controle da filariose linfática no $\mathrm{Mu}$ nicípio de Jaboatão dos Guararapes, norteando as atividades do plano nacional de eliminação da filariose linfática (MS, 1997). Defende-se aqui que a distribuição da filariose expressa a iniqüidade e ao mesmo tempo em que localiza os grupos prioritários em espaços determinados, auxiliando no planejamento do controle da endemia. Outro aspecto relevante é seu reconhecimento como a primeira etapa para definição de áreas de risco, que norteará as estratégias de intervenção, possibilitando ações setoriais específicas capazes de reduzir o sofrimento da população exposta a filariose. 


\section{Agradecimentos}

Agradecemos o apoio financeiro do Ministério da Saúde e da Coordenação de Aperfeiçoamento de Pessoal de Nível Superior. À Secretaria Municipal de Saúde de Jaboatão dos Guararapes: Dra. Lucia Vasconcelos (Diretora de Epidemiologia) e Dra. Vânia Benigno (Coordenação do Programa de Controle de Endemias). Aos agentes de saúde pela contribuição dada no inquérito epidemiológico. Aos técnicos do laboratório de parasitologia pela realização dos exames. Aos motoristas Marcos e Fernando pelo trabalho realizado na pesquisa.

\section{Referências}

ALBUQUERQUE, M. F. P. M., 1993. Urbanização, favelas e endemias: A produção da filariose no Recife, Brasil. Cadernos de Saúde Pública, 9:487-497.

ALBUQUERQUE, M. F. M.; MARZOCHI, M. C.; SABROZA, P. C.; BRAGA, M. C.; PADILHA, T.; SILVA, M. C. M.; SILVA, M. R. F.; SCHINDLER, H. C.; MACIEL, M. A.; SOUZA, W. \& FURTADO, A. F., 1995. Bancroftian filariasis in two urban áreas of Recife, Brazil: Pre-control observations on infection and disease. Transactions of the Royal Society of Tropical Medicine and Hygiene, 89:319-321.

AMARAL, F.; DREYER, G.; FIGUEREDO-SILVA, J.; NORÕES, J.; CAVALCANTI, A.; SAMICO, S. C.; SANTOS, A. \& COUTINHO, A., 1994. Live adult worms detected by ultrasonography in humam bancroftina filariasis. American Journal of Tropical Medicine and Hygiene, 50:753-757.

AZEVEDO, R. \& DOBBIN Jr., J. E., 1952. Filariose ( $W u$ chereria bancrofti) no grupo residencial do IAPB no bairro dos Afogados (Recife). In: Publicações Avulsas do Instituto Aggeu Magalhães, v. 1, n. 13, pp. 157-162, Recife: Instituto Aggeu Magalhães.

BRABIN, L., 1990. Sex differentials in susceptibility to lymphatic filariasis and implications for maternal child immunity. Epidemiology and Infections, 105:335-353.

DAS, P. K.; MANOOHARAN, A.; SRIVIDYA, A.; GRENFELL, B. T.; BUNDY, D. A. P. \& VANAMAIL, P., 1990. Frequency distribution of Wuchereria bancrofti microfilárias in human populations and its relationships with age and sex. Parasitology, 101:429434.

DEAN, M., 2000. At last, the fight against lymphatic filariasis begins. Lancet, 355:385.

DREYER, G. \& NORÕES, J., 1997a. Filariose bancroftiana. In: Condutas em Clínica Médica (V. G. Lucena, M. F. M. Albuquerque, T. A. A. Lima, T. F. G. Silva, C. C. S. Leitão, C. A. A. Brito, N. A. Figueira \& H. Ramos, org.), pp. 339-421, Recife: Editora Universitária.
DREYER, G. \& NORÕES, J., 1997b. Dietilcarbamazina no tratamento da filariose bancroftiana. Revista da Sociedade Brasileira de Medicina Tropical, 30:229-240.

DREYER, G.; PIMENTAEL, A.; MEDEIROS, Z.; BÉLIZ, F.; MOURA, I.; COUTINHO, A.; ANDRADE, L. D.; ROCHA, A.; DA SILVA, L. M. \& PIESSENS, W. F., 1996. Studies on the periodicity and intravascular distribuiton of Wuchereria bancrofti microfilariae in paired samples of capillary and venous blood from Recife, Brazil. Tropical Medicine and International Health, 1:264-272.

DUNYO, S. K.; APPAWU, M.; NKRUMAH, F. K.; BAFFOE-WILMONT, A.; PEDERSON, E. M. \& SIMONSEN, P. E., 1996. Lymphatic filariasis of the coast of Ghana. Transactions of the Royal Society of Tropical Medicine and Hygiene, 90:634-638.

FRANCO, O. \& LIMA, D. M. S., 1967. Alguns aspectos das atividades contra a filariose bancroftiana no Brasil. Revista Brasileira de Malariologia e Doenças Tropicais, 19:73-89.

IBGE (Fundação Instituto Brasileiro de Geografia e Estatística), 1996. Recontagem Populacional, 1996. Rio de Janeiro: IBGE.

MACIEL, M. A.; MARZOCHI, K. B.; SILVA, E. C.; ROCHA, A. \& FURTADO, A. F., 1994. Estudo comparativo de áreas endêmicas de filariose bancroftiana na Região Metropolitana do Recife, Brasil. Cadernos de Saúde Pública, 10(Sup. 2):301-309.

MEDEIROS, Z., 1998. Contribuições ao Estudo Epidemiológico da Filariose na Região Metropolitana do Recife. Tese de Doutorado, Rio de Janeiro: Instituto Oswaldo Cruz, Fundação Oswaldo Cruz.

MEDEIROS, Z.; DREYER, G.; ANDRADE, L.; PIRES, M. L.; MENDES, J. \& PIMENTEL, R., 1992. Wuchereria bancrofti microfilarial density of autochthonous cases and natural Culex infectivity in Northeast Brazil. Journal of Tropical Medicine and Hygiene, 95:214-217. 
MEDEIROS, Z.; GOMES, J.; BÉLIZ, F.; COUTINHO, A.; DREYER, P. \& DREYER, G., 1999. Screening of army soldiers for Wuchereria bancrofti infection in the metropolitan Recife region, Brazil: Implications for epidemiological surveillance. Tropical Medicine and International Health, 4:499-505.

MICHAEL, E. \& BUNDY, D. A. P., 1997. Global mapping of lymphatic filariasis. Parasitology Today, 13:472-476.

MICHAEL, E.; BUNDY, D. A. P. \& GRENFELL, B. T., 1996. Re-assessing the global prevalence and distribution of lymphatic filariasis. Parasitology, 112:409-428.

MS (Ministério da Saúde), 1985. Filarioses. In: O Controle das Endemias no Brasil (de 1979 a 1984) (MS, org.), pp. 130-133, Brasília: Superintendência de Campanhas de Saúde Pública, Ministério da Saúde.

MS (Ministério da Saúde), 1997. Programa de Eliminação da Filariose Linfática no Brasil. Brasília: Coordenação do Controle de Doenças Transmitidas por Vetores, Gerência de Endemias Focais, Fundação Nacional de Saúde.

MS (Ministério da Saúde), 2000. Reunião de Avaliação do Programa de Controle da Filariose Linfática no Brasil. Brasília: Centro Nacional de Epidemiologia.

OMS (Organización Mundial de la Salud), 1988. $L u$ cha Contra la Filariasis Linfática. Geneva: OMS.

PANI, S. P.; BALAKRISNAM, N.; SRIVIDYA, A.; BUNDY, D. A. \& GRENFELL, B. T., 1991. Clinical epidemiology of bancroftian filariasis: Effect of age and gender. Transactions of the Royal Society of Tropical Medicine and Hygiene, 85:260-264.
RACHOU, R., 1957. Distribuição geográfica das filarioses humanas no Brasil. Revista Brasileira de Malariologia e Doenças Tropicais, 9:79-100.

RACHOU, R., 1960. Conceito e programa de profilaxia da filariose bancroftiana no Brasil. Revista Brasileira de Malariologia e Doenças Tropicais, 12:1139.

RACHOU, R.; VILLELA, A. M.; CRUZ, A. E. \& CARVALHO, G. A., 1956. Filariose bancroftiana em Recife (Pernambuco). Resultado de um inquérito realizado em 1954-1955. Revista Brasileira de Malariologia e Doenças Tropicais, 8:359-367.

RAJASEKARIAH, G. R.; PARAB, P. B.; CHANDRASHEKAR, R.; DESHPANDE, L. \& SUBRAHMNYAM, D., 1991. Pattern of Wuchereria bancrofti mocrofilareamia in young and adolescent scholl children in Bassein, India, na endemic area lymphatic filariasis. Annals of Tropical Medicine and Parasitology, 85:663-665.

WHO (World Health Organization), 1994. Lymphatic Filariasis Infection \& Disease. Control Strategies. Report of a consultative meeting at the Universiti Sains Malaysia. Geneva: WHO.

WHO (World Health Organization), 2000. Eliminate Filariasis: Attack Poverty. Proceedings of the First Meeting of the Global Alliance to Eliminate Lymphatic Filariasis. Geneva: WHO.

Recebido em 3 de janeiro de 2003

Versão final reapresentada em 15 de abril de 2003

Aprovado em 10 de julho de 2003 